\title{
Quantum Particle Production in Cosmology
}

\author{
Ruth Durrer
}

Université de Genève - Genève - France 


\title{
Quantum Particle Production in Cosmology
}

\author{
Ruth Durrer
}

Département de physique théorique, Université de Genève

\section{UNIVERSITÉ} DE GENĖVE

QED and Quantum Vacuum Low Energy Frontier

Cargèse, April 2012 


\section{Outline}

(1) Introduction to cosmology

(2) Quantum fluctuations of the inflaton

(3) Quantum fluctuations of the graviton

(4) The post-inflationary Universe

(5) conclusions 


\section{Introduction: The Friedmann-Lemaître universe}

The observed Universe is very isotropic and homogeneous on large scales. On large scale its geometry is well approximated by an expanding Friedmann-Lemaître Universe,

$$
d s^{2}=-d t^{2}+a^{2}(t) \gamma_{i j} d x^{i} d x^{j}
$$

where $\gamma$ is the metric of a 3-space of constant curvature $K$ and the scale factor $a(t)$ describes the expansion of the Universe. 


\section{Introduction: The Friedmann-Lemaître universe}

The observed Universe is very isotropic and homogeneous on large scales. On large scale its geometry is well approximated by an expanding Friedmann-Lemaître Universe,

$$
d s^{2}=-d t^{2}+a^{2}(t) \gamma_{i j} d x^{i} d x^{j}
$$

where $\gamma$ is the metric of a 3-space of constant curvature $K$ and the scale factor $a(t)$ describes the expansion of the Universe.

The rate of expansion, $\dot{a} / a$ and the deceleration/acceleration are related to the energy density of the Universe via Einstein's equation (called Friemann equations in this context).

$$
\left(\frac{\dot{a}}{a}\right)^{2}+\frac{K}{a^{2}}=\frac{8 \pi G}{3} \rho+\frac{\Lambda}{3}, \quad \frac{\ddot{a}}{a}=-\frac{4 \pi G}{3}(\rho+3 P)+\frac{\Lambda}{3}
$$




\section{Introduction: The Friedmann-Lemaître universe}

The observed Universe is very isotropic and homogeneous on large scales. On large scale its geometry is well approximated by an expanding Friedmann-Lemaître Universe,

$$
d s^{2}=-d t^{2}+a^{2}(t) \gamma_{i j} d x^{i} d x^{j}
$$

where $\gamma$ is the metric of a 3-space of constant curvature $K$ and the scale factor $a(t)$ describes the expansion of the Universe.

The rate of expansion, $\dot{a} / a$ and the deceleration/acceleration are related to the energy density of the Universe via Einstein's equation (called Friemann equations in this context).

$$
\left(\frac{\dot{a}}{a}\right)^{2}+\frac{K}{a^{2}}=\frac{8 \pi G}{3} \rho+\frac{\Lambda}{3}, \quad \frac{\ddot{a}}{a}=-\frac{4 \pi G}{3}(\rho+3 P)+\frac{\Lambda}{3}
$$

We measure expansion in terms of redshift $z(t)+1=a\left(t_{0}\right) / a(t)=1 / a(t)$. 


\section{Introduction: The Friedmann-Lemaître universe}

The observed Universe is very isotropic and homogeneous on large scales. On large scale its geometry is well approximated by an expanding Friedmann-Lemaître Universe,

$$
d s^{2}=-d t^{2}+a^{2}(t) \gamma_{i j} d x^{i} d x^{j}
$$

where $\gamma$ is the metric of a 3-space of constant curvature $K$ and the scale factor $a(t)$ describes the expansion of the Universe.

The rate of expansion, $\dot{a} / a$ and the deceleration/acceleration are related to the energy density of the Universe via Einstein's equation (called Friemann equations in this context).

$$
\left(\frac{\dot{a}}{a}\right)^{2}+\frac{K}{a^{2}}=\frac{8 \pi G}{3} \rho+\frac{\Lambda}{3}, \quad \frac{\ddot{a}}{a}=-\frac{4 \pi G}{3}(\rho+3 P)+\frac{\Lambda}{3}
$$

We measure expansion in terms of redshift $z(t)+1=a\left(t_{0}\right) / a(t)=1 / a(t)$. We measure energy densities, cosmological constant, curvature in terms of their contribution to the expansion rate $H \equiv \dot{a} / a$.

$$
\Omega_{X}=\frac{8 \pi G \rho_{X}}{3 H^{2}}, \quad \Omega_{\Lambda}=\frac{\Lambda}{3 H^{2}}, \quad \Omega_{K}=-\frac{K}{a^{2} H^{2}},
$$




\section{Introduction: The Friedmann-Lemaître universe}

The observed Universe is very isotropic and homogeneous on large scales. On large scale its geometry is well approximated by an expanding Friedmann-Lemaître Universe,

$$
d s^{2}=-d t^{2}+a^{2}(t) \gamma_{i j} d x^{i} d x^{j}
$$

where $\gamma$ is the metric of a 3-space of constant curvature $K$ and the scale factor $a(t)$ describes the expansion of the Universe.

The rate of expansion, $\dot{a} / a$ and the deceleration/acceleration are related to the energy density of the Universe via Einstein's equation (called Friemann equations in this context).

$$
\left(\frac{\dot{a}}{a}\right)^{2}+\frac{K}{a^{2}}=\frac{8 \pi G}{3} \rho+\frac{\Lambda}{3}, \quad \frac{\ddot{a}}{a}=-\frac{4 \pi G}{3}(\rho+3 P)+\frac{\Lambda}{3}
$$

We measure expansion in terms of redshift $z(t)+1=a\left(t_{0}\right) / a(t)=1 / a(t)$.

We measure energy densities, cosmological constant, curvature in terms of their contribution to the expansion rate $H \equiv \dot{a} / a$.

$$
\Omega_{X}=\frac{8 \pi G \rho_{X}}{3 H^{2}}, \quad \Omega_{\Lambda}=\frac{\Lambda}{3 H^{2}}, \quad \Omega_{K}=-\frac{K}{a^{2} H^{2}},
$$

At present $\Omega_{m} \simeq 0.3, \Omega_{b} \simeq 0.04, \Omega_{\gamma} \simeq 10^{-4}, 0.03>\Omega_{\nu}>0.003, \Omega_{\Lambda} \simeq 0.7, \Omega_{K} \simeq 0$. If $\Lambda=0$ and $P>-\rho / 3, \Omega_{K}=0$ is an unstable fix point of cosmic expansion. 


\section{Introduction: The CMB}

In the past, the Universe was not only much denser but also much hotter... 


\section{Introduction: The CMB}

In the past, the Universe was not only much denser but also much hotter...

At a temperature of about $T \simeq 3000 \mathrm{~K}, z \simeq 1100, t \simeq 3 \times 10^{5}$ years, protons and electrons combined to neutral hydrogen and the Universe became transparent to photons. Today we see these photons in the cosmic microwave background (CMB) which has a perfect Planck spectrum with temperature $T=2.7255 \pm 0.0006 \mathrm{~K}$.

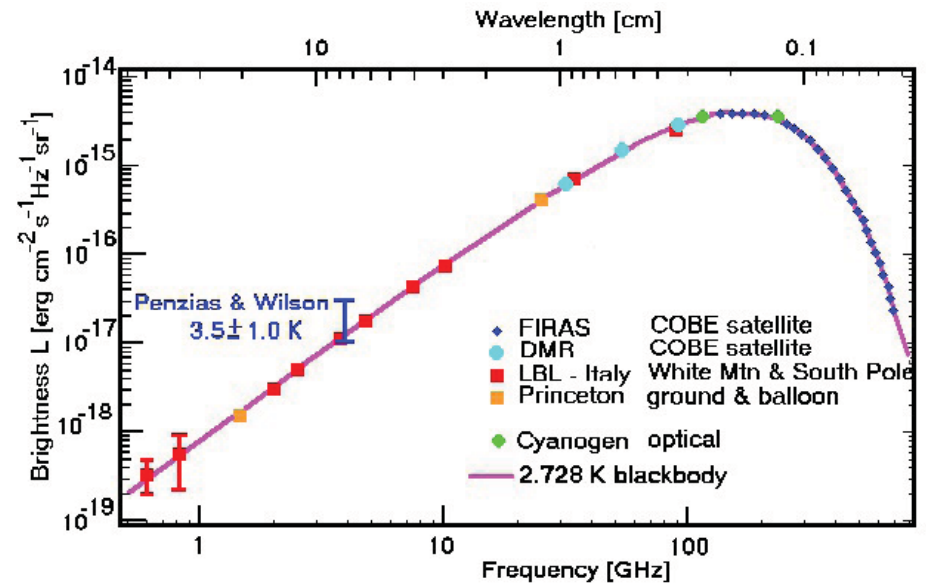




\section{Introduction: Thermal history}

- Before recombination, at $z \simeq 3000$ the energy density in matter equaled that in radiation. 


\section{Introduction: Thermal history}

- Before recombination, at $z \simeq 3000$ the energy density in matter equaled that in radiation.

- At $z \simeq 4 \times 10^{7}, T \simeq 0.1 \mathrm{MeV}, t \simeq 200$ s the neutrons remaining in the Universe are bound mainly to $\mathrm{He}^{4}$ leading to a Helium abundance of about $23 \%$.

Nucleosynthesis 


\section{Introduction: Thermal history}

- Before recombination, at $z \simeq 3000$ the energy density in matter equaled that in radiation.

- At $z \simeq 4 \times 10^{7}, T \simeq 0.1 \mathrm{MeV}, t \simeq 200$ s the neutrons remaining in the Universe are bound mainly to $\mathrm{He}^{4}$ leading to a Helium abundance of about $23 \%$. Nucleosynthesis

- At $z \simeq 6 \times 10^{8}, T \simeq 1.4 \mathrm{MeV}, t \simeq 1$ neutrinos decouple. Leading to a cosmic neutrino background which at present should have a temperature $T_{\nu}=1.7 \mathrm{~K}$. 


\section{Introduction: Thermal history}

- Before recombination, at $z \simeq 3000$ the energy density in matter equaled that in radiation.

- At $z \simeq 4 \times 10^{7}, T \simeq 0.1 \mathrm{MeV}, t \simeq 200$ s the neutrons remaining in the Universe are bound mainly to $\mathrm{He}^{4}$ leading to a Helium abundance of about $23 \%$. Nucleosynthesis

- At $z \simeq 6 \times 10^{8}, T \simeq 1.4 \mathrm{MeV}, t \simeq 1$ neutrinos decouple. Leading to a cosmic neutrino background which at present should have a temperature $T_{\nu}=1.7 \mathrm{~K}$.

- At $z \simeq 4 \times 10^{10}, T \simeq 100 \mathrm{MeV}, t \simeq 10^{-3}$ s quarks bind into protons and neutrons. Confinement 


\section{Introduction: Thermal history}

- Before recombination, at $z \simeq 3000$ the energy density in matter equaled that in radiation.

- At $z \simeq 4 \times 10^{7}, T \simeq 0.1 \mathrm{MeV}, t \simeq 200$ s the neutrons remaining in the Universe are bound mainly to $\mathrm{He}^{4}$ leading to a Helium abundance of about $23 \%$. Nucleosynthesis

- At $z \simeq 6 \times 10^{8}, T \simeq 1.4 \mathrm{MeV}, t \simeq 1$ neutrinos decouple. Leading to a cosmic neutrino background which at present should have a temperature $T_{\nu}=1.7 \mathrm{~K}$.

- At $z \simeq 4 \times 10^{10}, T \simeq 100 \mathrm{MeV}, t \simeq 10^{-3} \mathrm{~s}$ quarks bind into protons and neutrons. Confinement

- At $z \simeq 4 \times 10^{13}, T \simeq 100 \mathrm{GeV}, t \simeq 10^{-9}$ s electroweak transition. 


\section{Introduction: Thermal history}

- Before recombination, at $z \simeq 3000$ the energy density in matter equaled that in radiation.

- At $z \simeq 4 \times 10^{7}, T \simeq 0.1 \mathrm{MeV}, t \simeq 200$ s the neutrons remaining in the Universe are bound mainly to $\mathrm{He}^{4}$ leading to a Helium abundance of about $23 \%$. Nucleosynthesis

- At $z \simeq 6 \times 10^{8}, T \simeq 1.4 \mathrm{MeV}, t \simeq 1$ neutrinos decouple. Leading to a cosmic neutrino background which at present should have a temperature $T_{\nu}=1.7 \mathrm{~K}$.

- At $z \simeq 4 \times 10^{10}, T \simeq 100 \mathrm{MeV}, t \simeq 10^{-3} \mathrm{~s}$ quarks bind into protons and neutrons. Confinement

- At $z \simeq 4 \times 10^{13}, T \simeq 100 \mathrm{GeV}, t \simeq 10^{-9}$ s electroweak transition. 


\section{Introduction: Thermal history}

\section{Big Bang}

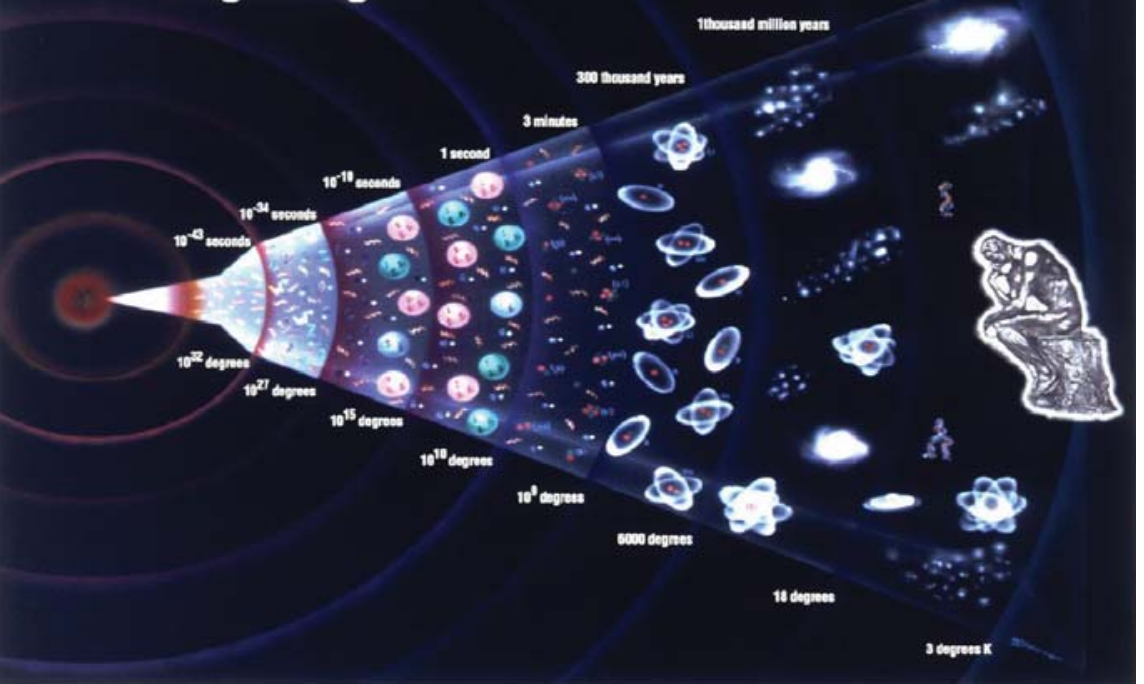




\section{Introduction: Cosmic structure}

- The Universe around us is not homogeneous and isotropic. It contains galaxies, clusters, filaments, voids...

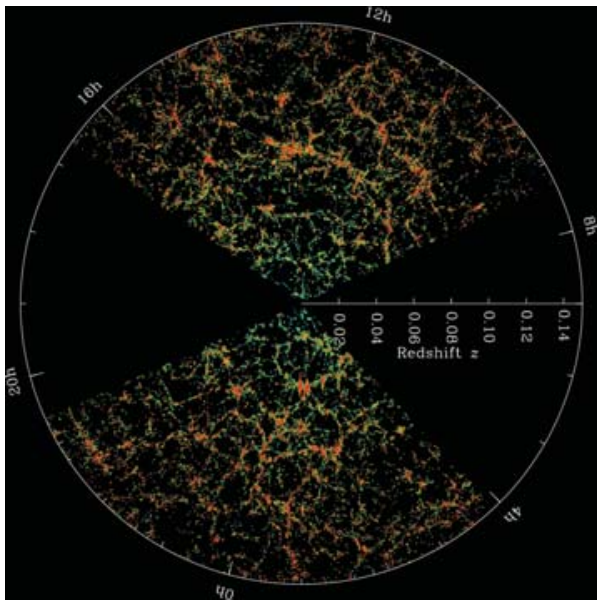

From the Sloan Digital Sky Survey 


\section{Introduction: Cosmic structure}

- The Universe around us is not homogeneous and isotropic. It contains galaxies, clusters, filaments, voids...

- The idea is that these have grown out of small initial fluctuations by gravitational instability. In an expanding universe gravitational instability is not exponential but only a power law. 


\section{Introduction: Cosmic structure}

- The Universe around us is not homogeneous and isotropic. It contains galaxies, clusters, filaments, voids...

- The idea is that these have grown out of small initial fluctuations by gravitational instability. In an expanding universe gravitational instability is not exponential but only a power law.

- Actually, as long a the Universe is radiation dominated, radiation pressure completely prevents the growth of structure. Once, the Universe becomes matter dominated density fluctuations start growing $\propto a$, but within linear perturbation theory the gravitational potential remains constant. 
- The Universe around us is not homogeneous and isotropic. It contains galaxies, clusters, filaments, voids...

- The idea is that these have grown out of small initial fluctuations by gravitational instability. In an expanding universe gravitational instability is not exponential but only a power law.

- Actually, as long a the Universe is radiation dominated, radiation pressure completely prevents the growth of structure. Once, the Universe becomes matter dominated density fluctuations start growing $\propto$ a, but within linear perturbation theory the gravitational potential remains constant.

- For present structure to develop, initial perturbations of an amplitude $\Psi \simeq 3 \times 10^{-5}$ are needed. 
- The Universe around us is not homogeneous and isotropic. It contains galaxies, clusters, filaments, voids...

- The idea is that these have grown out of small initial fluctuations by gravitational instability. In an expanding universe gravitational instability is not exponential but only a power law.

- Actually, as long a the Universe is radiation dominated, radiation pressure completely prevents the growth of structure. Once, the Universe becomes matter dominated density fluctuations start growing $\propto a$, but within linear perturbation theory the gravitational potential remains constant.

- For present structure to develop, initial perturbations of an amplitude $\psi \simeq 3 \times 10^{-5}$ are needed.

- This corresponds exactly to the fluctuations observed in the cosmic microwave background. 


\section{Introduction: Cosmic structure}

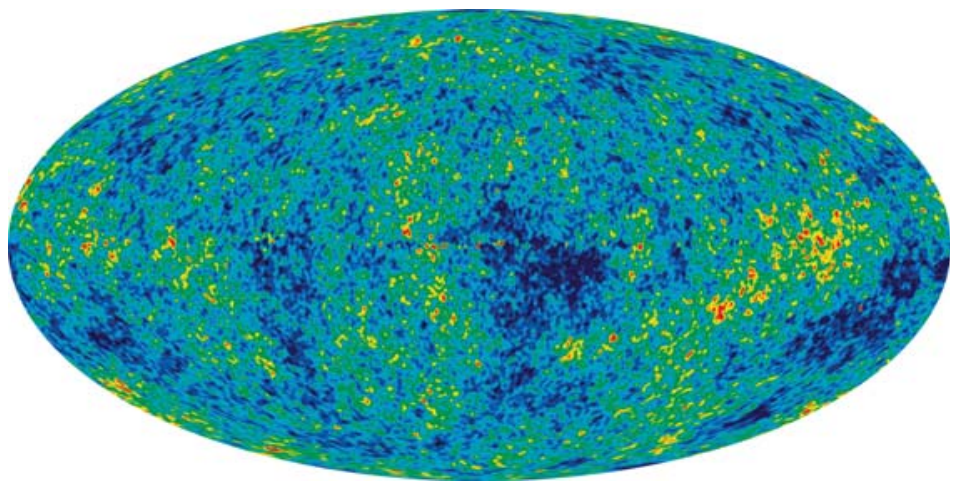

CMB anisotropies (WMAP 7 year data) 


\section{Inflation}

- Why is curvature so small, $|K| / H^{2}(t)=|\Omega(t)-1|<0.01$. If we scale a radiation dominated universe back to Planck time, $\left|\Omega\left(t_{P I}\right)-1\right|<10^{-60}$ ? 


\section{Inflation}

- Why is curvature so small, $|K| / H^{2}(t)=|\Omega(t)-1|<0.01$. If we scale a radiation dominated universe back to Planck time, $\left|\Omega\left(t_{P I}\right)-1\right|<10^{-60}$ ?

- Why is the entropy per baryon so large $\left(\simeq 10^{10}\right)$ ? 


\section{Inflation}

- Why is curvature so small, $|K| / H^{2}(t)=|\Omega(t)-1|<0.01$. If we scale a radiation dominated universe back to Planck time, $\left|\Omega\left(t_{P I}\right)-1\right|<10^{-60}$ ?

- Why is the entropy per baryon so large $\left(\simeq 10^{10}\right)$ ?

- How can one dilute 'unwanted relics' like monopoles which form in most theories of very high energy physics (e.g. GUTs)? 


\section{Inflation}

- Why is curvature so small, $|K| / H^{2}(t)=|\Omega(t)-1|<0.01$. If we scale a radiation dominated universe back to Planck time, $\left|\Omega\left(t_{P I}\right)-1\right|<10^{-60}$ ?

- Why is the entropy per baryon so large $\left(\simeq 10^{10}\right)$ ?

- How can one dilute 'unwanted relics' like monopoles which form in most theories of very high energy physics (e.g. GUTs)?

- How are the initial fluctuations generated? 


\section{Inflation}

- Why is curvature so small, $|K| / H^{2}(t)=|\Omega(t)-1|<0.01$. If we scale a radiation dominated universe back to Planck time, $\left|\Omega\left(t_{P I}\right)-1\right|<10^{-60}$ ?

- Why is the entropy per baryon so large $\left(\simeq 10^{10}\right)$ ?

- How can one dilute 'unwanted relics' like monopoles which form in most theories of very high energy physics (e.g. GUTs)?

- How are the initial fluctuations generated?

- Inflation is a phase of very rapid, accelerated cosmological expansion which has been invented to solve the first 3 problems A. Guth, 1981. 


\section{Inflation}

- Why is curvature so small, $|K| / H^{2}(t)=|\Omega(t)-1|<0.01$. If we scale a radiation dominated universe back to Planck time, $\left|\Omega\left(t_{P I}\right)-1\right|<10^{-60}$ ?

- Why is the entropy per baryon so large $\left(\simeq 10^{10}\right)$ ?

- How can one dilute 'unwanted relics' like monopoles which form in most theories of very high energy physics (e.g. GUTs)?

- How are the initial fluctuations generated?

- Inflation is a phase of very rapid, accelerated cosmological expansion which has been invented to solve the first 3 problems A. Guth, 1981.

- In GR the 'the gravitational force' is proportional to $4 \pi G(\rho+3 P)$ If $\rho+3 P<0$ gravity becomes repulsive and the expansion of the Universe is accelerated. 
- Why is curvature so small, $|K| / H^{2}(t)=|\Omega(t)-1|<0.01$. If we scale a radiation dominated universe back to Planck time, $\left|\Omega\left(t_{P I}\right)-1\right|<10^{-60}$ ?

- Why is the entropy per baryon so large $\left(\simeq 10^{10}\right)$ ?

- How can one dilute 'unwanted relics' like monopoles which form in most theories of very high energy physics (e.g. GUTs)?

- How are the initial fluctuations generated?

- Inflation is a phase of very rapid, accelerated cosmological expansion which has been invented to solve the first 3 problems A. Guth, 1981.

- In GR the 'the gravitational force' is proportional to $4 \pi G(\rho+3 P)$ If $\rho+3 P<0$ gravity becomes repulsive and the expansion of the Universe is accelerated.

- The simplest way to obtain accelerated expansion is a universe dominated by a scalar field, $\phi$ where $\rho+3 P=\dot{\phi}^{2}-2 V$ becomes negative if the potential dominates over the kinetic term. 
- Why is curvature so small, $|K| / H^{2}(t)=|\Omega(t)-1|<0.01$. If we scale a radiation dominated universe back to Planck time, $\left|\Omega\left(t_{P I}\right)-1\right|<10^{-60}$ ?

- Why is the entropy per baryon so large $\left(\simeq 10^{10}\right)$ ?

- How can one dilute 'unwanted relics' like monopoles which form in most theories of very high energy physics (e.g. GUTs)?

- How are the initial fluctuations generated?

- Inflation is a phase of very rapid, accelerated cosmological expansion which has been invented to solve the first 3 problems A. Guth, 1981.

- In GR the 'the gravitational force' is proportional to $4 \pi G(\rho+3 P)$ If $\rho+3 P<0$ gravity becomes repulsive and the expansion of the Universe is accelerated.

- The simplest way to obtain accelerated expansion is a universe dominated by a scalar field, $\phi$ where $\rho+3 P=\dot{\phi}^{2}-2 V$ becomes negative if the potential dominates over the kinetic term.

- It has been clear already at this time, that an inflationary phase also generates a nearly scale invariant spectrum of scalar V. Mukhanov \& G. Chibisov, 1981 and tensor A. Starobinski, 1979 fluctuations. 


\section{Inflation}

- As we shall see, the scalar fluctations are quantum fluctuations of the inflaton field which are generated by its coupling to a time dependent classical field: the expansion of the universe. 


\section{Inflation}

- As we shall see, the scalar fluctations are quantum fluctuations of the inflaton field which are generated by its coupling to a time dependent classical field: the expansion of the universe.

- Equivalently, the tensor fluctuations are quantum fluctuations of the metric. Their detection would be a proof of the quantum nature of spacetime geometry! 


\section{Inflation}

- As we shall see, the scalar fluctations are quantum fluctuations of the inflaton field which are generated by its coupling to a time dependent classical field: the expansion of the universe.

- Equivalently, the tensor fluctuations are quantum fluctuations of the metric. Their detection would be a proof of the quantum nature of spacetime geometry!

- After inflation the scalar field energy is converted into heat (relativistic particles in thermal equilibrium) thereby generating a large amount of entropy. 
- As we shall see, the scalar fluctations are quantum fluctuations of the inflaton field which are generated by its coupling to a time dependent classical field: the expansion of the universe.

- Equivalently, the tensor fluctuations are quantum fluctuations of the metric. Their detection would be a proof of the quantum nature of spacetime geometry!

- After inflation the scalar field energy is converted into heat (relativistic particles in thermal equilibrium) thereby generating a large amount of entropy.

- The details of this process of pre-heating and reheating are very model dependent, i.e. they depend on the coupling of the inflaton field to other degrees of freedom and to standard model particles. 


\section{Inflation}

- After inflation the fluctuations of the inflaton field become classical fluctuations in the energy density end in the gravitational potential. The tensor fluctuations become classical gravitational waves, i.e. classical tensor fluctuations of the metric. 


\section{Inflation}

- After inflation the fluctuations of the inflaton field become classical fluctuations in the energy density end in the gravitational potential. The tensor fluctuations become classical gravitational waves, i.e. classical tensor fluctuations of the metric.

- The scalar and tensor fluctuations are expected to be (nearly) Gaussian and hence characterized by their power spectra, $P_{S}(k)$ and $P_{T}(k)$ which can be computed in a given inflationary model.

$$
\begin{gathered}
k^{3}\left\langle\Psi(\mathbf{k}) \Psi^{*}\left(\mathbf{k}^{\prime}\right)\right\rangle=(2 \pi)^{3} \delta\left(\mathbf{k}-\mathbf{k}^{\prime}\right) P_{S}(k), \\
k^{3}\left\langle h(\mathbf{k}) h^{*}\left(\mathbf{k}^{\prime}\right)\right\rangle=(2 \pi)^{3} \delta\left(\mathbf{k}-\mathbf{k}^{\prime}\right) P_{T}(k) .
\end{gathered}
$$




\section{CMB anisotropies}

Anisotropies in the CMB are characterized by their angular power spectrum defined as

$$
\left\langle\frac{\delta T(\mathbf{n})}{T} \frac{\delta T\left(\mathbf{n}^{\prime}\right)}{T}\right\rangle=\frac{1}{4 \pi} \sum_{\ell}(2 \ell+1) C_{\ell} P_{\ell}\left(\mathbf{n} \cdot \mathbf{n}^{\prime}\right) .
$$




\section{CMB anisotropies}

Anisotropies in the CMB are characterized by their angular power spectrum defined as

$$
\left\langle\frac{\delta T(\mathbf{n})}{T} \frac{\delta T\left(\mathbf{n}^{\prime}\right)}{T}\right\rangle=\frac{1}{4 \pi} \sum_{\ell}(2 \ell+1) C_{\ell} P_{\ell}\left(\mathbf{n} \cdot \mathbf{n}^{\prime}\right) .
$$

After inflation, the evolution of the initial fluctuations can be computed by classical linear cosmological perturbation theory and transfer functions which relate them to the angular power spectrum of the CMB:

$$
C_{\ell}=4 \pi \int \frac{d k}{k}\left[T_{S}(\ell, k) P_{S}(k)+T_{T}(\ell, k) P_{T}(k)\right]
$$




\section{CMB anisotropies}

Anisotropies in the CMB are characterized by their angular power spectrum defined as

$$
\left\langle\frac{\delta T(\mathbf{n})}{T} \frac{\delta T\left(\mathbf{n}^{\prime}\right)}{T}\right\rangle=\frac{1}{4 \pi} \sum_{\ell}(2 \ell+1) C_{\ell} P_{\ell}\left(\mathbf{n} \cdot \mathbf{n}^{\prime}\right) .
$$

After inflation, the evolution of the initial fluctuations can be computed by classical linear cosmological perturbation theory and transfer functions which relate them to the angular power spectrum of the CMB:

$$
C_{\ell}=4 \pi \int \frac{d k}{k}\left[T_{S}(\ell, k) P_{S}(k)+T_{T}(\ell, k) P_{T}(k)\right]
$$

The transfer functions $T_{S}(\ell, k)$ and $T_{T}(\ell, k)$ depend on the cosmological parameters. 


\section{CMB anisotropies}

Anisotropies in the CMB are characterized by their angular power spectrum defined as

$$
\left\langle\frac{\delta T(\mathbf{n})}{T} \frac{\delta T\left(\mathbf{n}^{\prime}\right)}{T}\right\rangle=\frac{1}{4 \pi} \sum_{\ell}(2 \ell+1) C_{\ell} P_{\ell}\left(\mathbf{n} \cdot \mathbf{n}^{\prime}\right) .
$$

After inflation, the evolution of the initial fluctuations can be computed by classical linear cosmological perturbation theory and transfer functions which relate them to the angular power spectrum of the CMB:

$$
C_{\ell}=4 \pi \int \frac{d k}{k}\left[T_{S}(\ell, k) P_{S}(k)+T_{T}(\ell, k) P_{T}(k)\right]
$$

The transfer functions $T_{S}(\ell, k)$ and $T_{T}(\ell, k)$ depend on the cosmological parameters. If the inflationary spectra are simple (described by few parameters, e.g.

$$
P_{S}(k)=A_{s}\left(k / k_{*}\right)^{n_{s}-1} \quad P_{S}(k)=A_{t}\left(k / k_{*}\right)^{n_{t}}
$$

The observations can be used to determine the cosmological parameters. 


\section{Introduction: CMB anisotropies}
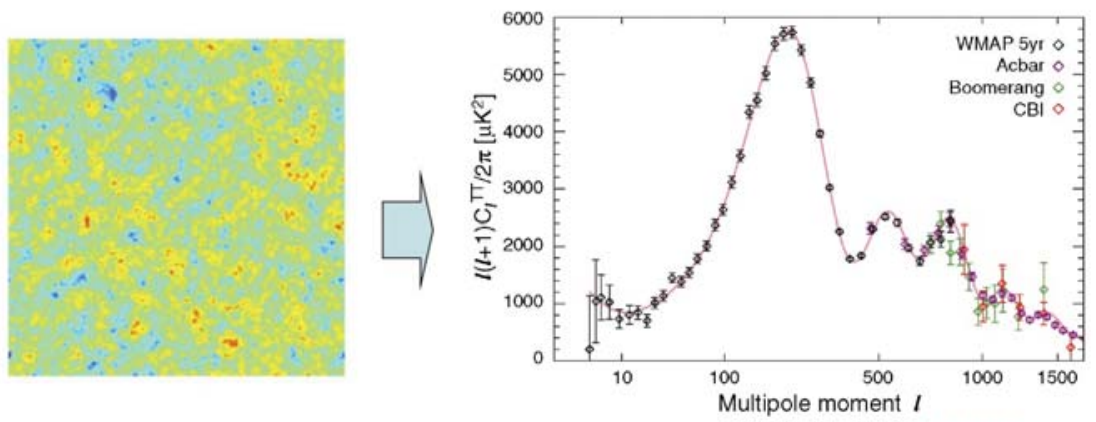

WMAP team

$$
\begin{aligned}
& n_{s}=0.96, A_{s}=2.4 \times 10^{-9}, r=A_{t} / A_{s} \\
& \Omega_{m} h^{2}=0.12, \Omega_{b} h^{2}=0.023, \tau=0.088, H_{0}=71.4 \mathrm{~km} / \mathrm{sec} / \mathrm{Mpc}=h 100 \mathrm{~km} / \mathrm{sec} / \mathrm{Mpc} \\
& K=0, \Omega_{\Lambda}=1-\Omega_{m}
\end{aligned}
$$




\section{Quantum fluctuations of the inflaton I}

The metric of a homogeneous and isotropic Universe is of the form

$$
d s^{2}=-d t^{2}+a^{2}(t) \gamma_{i j} d x^{i} d x^{j} .
$$

$H(t)=\dot{a} / a$ is the Hubble parameter. 


\section{Quantum fluctuations of the inflaton I}

The metric of a homogeneous and isotropic Universe is of the form

$$
d s^{2}=-d t^{2}+a^{2}(t) \gamma_{i j} d x^{i} d x^{j} .
$$

$H(t)=\dot{a} / a$ is the Hubble parameter.

Coupling a quantum field to a time dependent external classical field can lead to particle creation. The energy of the modes which can be generated is determined by the kinetic energy in the time dependence of the external field. In an expanding universe, this is determined by the Hubble parameter $H(t)$ which is nearly constant during inflation,

$$
H^{2}=\frac{8 \pi G}{3}\left(\dot{\phi}^{2}+V(\phi)\right) \simeq \frac{8 \pi G}{3} V, \quad \dot{\phi}^{2}=\frac{2}{3} \epsilon V \ll V(\phi) \quad \text { (slow roll) }
$$




\section{Quantum fluctuations of the inflaton I}

The metric of a homogeneous and isotropic Universe is of the form

$$
d s^{2}=-d t^{2}+a^{2}(t) \gamma_{i j} d x^{i} d x^{j} .
$$

$H(t)=\dot{a} / a$ is the Hubble parameter.

Coupling a quantum field to a time dependent external classical field can lead to particle creation. The energy of the modes which can be generated is determined by the kinetic energy in the time dependence of the external field. In an expanding universe, this is determined by the Hubble parameter $H(t)$ which is nearly constant during inflation,

$$
H^{2}=\frac{8 \pi G}{3}\left(\dot{\phi}^{2}+V(\phi)\right) \simeq \frac{8 \pi G}{3} V, \quad \dot{\phi}^{2}=\frac{2}{3} \epsilon V \ll V(\phi) \quad \text { (slow roll) }
$$

We set the inflaton field as $\phi=\varphi+\delta \phi$, where $\varphi$ is the classical background field (a condensate) which drives inflation and $\delta \phi$ is a small quantum fluctuation. 


\section{Quantum fluctuations of the inflaton I}

The metric of a homogeneous and isotropic Universe is of the form

$$
d s^{2}=-d t^{2}+a^{2}(t) \gamma_{i j} d x^{i} d x^{j} .
$$

$H(t)=\dot{a} / a$ is the Hubble parameter.

Coupling a quantum field to a time dependent external classical field can lead to particle creation. The energy of the modes which can be generated is determined by the kinetic energy in the time dependence of the external field. In an expanding universe, this is determined by the Hubble parameter $H(t)$ which is nearly constant during inflation,

$$
H^{2}=\frac{8 \pi G}{3}\left(\dot{\phi}^{2}+V(\phi)\right) \simeq \frac{8 \pi G}{3} V, \quad \dot{\phi}^{2}=\frac{2}{3} \epsilon V \ll V(\phi) \quad \text { (slow roll) }
$$

We set the inflaton field as $\phi=\varphi+\delta \phi$, where $\varphi$ is the classical background field (a condensate) which drives inflation and $\delta \phi$ is a small quantum fluctuation.

The small fluctuation in the inflaton also leads to small fluctuations in the geometry,

$$
d s^{2}=-(1+2 \Psi) d t^{2}+(1-2 \Psi) a^{2}(t) d \mathbf{x}^{2}
$$

Einstein's constraint equations relate $\Psi$ and $\delta \phi$ so that this system has only one degree of freedom. 


\section{Quantum fluctuations of the inflaton II}

Expanding the Lagrangian to 2 nd order in the fluctuation one can derive

$$
S=-\int d^{4} x \sqrt{\left|g_{b}\right|}\left(\frac{R_{b}}{16 \pi G}+L(\varphi)\right)+\delta S
$$




\section{Quantum fluctuations of the inflaton II}

Expanding the Lagrangian to 2 nd order in the fluctuation one can derive

$$
\begin{gathered}
S=-\int d^{4} x \sqrt{\left|g_{b}\right|}\left(\frac{R_{b}}{16 \pi G}+L(\varphi)\right)+\delta S \\
\delta S=-\frac{1}{2} \int d^{4} x\left(\partial_{\mu} v \partial^{\mu} v+m^{2}(t) v^{2}\right), \\
v=a(\delta \phi+(\dot{\varphi} / H) \Psi), m^{2}=\ddot{z} / z, z=\dot{\varphi} / H=\sqrt{\frac{\epsilon m_{P}^{2}}{4 \pi}}
\end{gathered}
$$




\section{Quantum fluctuations of the inflaton II}

Expanding the Lagrangian to 2 nd order in the fluctuation one can derive

$$
\begin{gathered}
S=-\int d^{4} x \sqrt{\left|g_{b}\right|}\left(\frac{R_{b}}{16 \pi G}+L(\varphi)\right)+\delta S \\
\delta S=-\frac{1}{2} \int d^{4} x\left(\partial_{\mu} v \partial^{\mu} v+m^{2}(t) v^{2}\right), \\
v=a(\delta \phi+(\dot{\varphi} / H) \Psi), m^{2}=\ddot{z} / z, z=\dot{\varphi} / H=\sqrt{\frac{\epsilon m_{P}^{2}}{4 \pi}}
\end{gathered}
$$

The canonically normalized field $v$ is simply related to the so called curvature fluctuation variable $\zeta \equiv v /(a z)$. 


\section{Quantum fluctuations of the inflaton II}

Expanding the Lagrangian to 2 nd order in the fluctuation one can derive

$$
\begin{gathered}
S=-\int d^{4} x \sqrt{\left|g_{b}\right|}\left(\frac{R_{b}}{16 \pi G}+L(\varphi)\right)+\delta S \\
\delta S=-\frac{1}{2} \int d^{4} x\left(\partial_{\mu} v \partial^{\mu} v+m^{2}(t) v^{2}\right), \\
v=a(\delta \phi+(\dot{\varphi} / H) \Psi), m^{2}=\ddot{z} / z, z=\dot{\varphi} / H=\sqrt{\frac{\epsilon m_{P}^{2}}{4 \pi}}
\end{gathered}
$$

The canonically normalized field $v$ is simply related to the so called curvature fluctuation variable $\zeta \equiv v /(a z)$. $d^{4} x=d \tau d^{3} x$, where $\tau$ is conformal time, $d \tau=d t / a$. 


\section{Quantum fluctuations of the inflaton II}

Expanding the Lagrangian to 2 nd order in the fluctuation one can derive

$$
\begin{gathered}
S=-\int d^{4} x \sqrt{\left|g_{b}\right|}\left(\frac{R_{b}}{16 \pi G}+L(\varphi)\right)+\delta S \\
\delta S=-\frac{1}{2} \int d^{4} x\left(\partial_{\mu} v \partial^{\mu} v+m^{2}(t) v^{2}\right), \\
v=a(\delta \phi+(\dot{\varphi} / H) \Psi), m^{2}=\ddot{z} / z, z=\dot{\varphi} / H=\sqrt{\frac{\epsilon m_{P}^{2}}{4 \pi}}
\end{gathered}
$$

The canonically normalized field $v$ is simply related to the so called curvature fluctuation variable $\zeta \equiv v /(a z)$. $d^{4} x=d \tau d^{3} x$, where $\tau$ is conformal time, $d \tau=d t / a$. During inflation $H$ is nearly constant, $a \propto \exp (H t)$,

$$
\tau=\int_{t_{i}}^{t} d t^{\prime} \exp \left(-H t^{\prime}\right) \simeq-H^{-1} \exp (-H t)
$$

is negative and $\tau \rightarrow 0$ when $t \rightarrow \infty$. 


\section{Quantum fluctuations of the inflaton III}

We now quantize the field $v$ and invoke the canonical commutation relations. We obtain the mode equation in Fourier space

$$
\frac{d^{2}}{d \tau^{2}} v_{k}+\left(k^{2}-\frac{2+9 \epsilon-3 \eta}{\tau^{2}}\right) v_{k}=0
$$




\section{Quantum fluctuations of the inflaton III}

We now quantize the field $v$ and invoke the canonical commutation relations. We obtain the mode equation in Fourier space

$$
\frac{d^{2}}{d \tau^{2}} v_{k}+\left(k^{2}-\frac{2+9 \epsilon-3 \eta}{\tau^{2}}\right) v_{k}=0
$$

Here $\epsilon$ and $\eta$ are the slow roll parameters which we assume to be small and slowly varying,

$$
\epsilon=\frac{1}{16 \pi G}\left(\frac{V_{, \varphi}}{V}\right)^{2} \ll 1, \eta=\frac{1}{24 \pi G}\left(\frac{V_{, \varphi \varphi}}{V}\right)=\frac{m_{P}^{2} m_{\varphi}^{2}}{3 V} \ll 1 .
$$




\section{Quantum fluctuations of the inflaton III}

We now quantize the field $v$ and invoke the canonical commutation relations. We obtain the mode equation in Fourier space

$$
\frac{d^{2}}{d \tau^{2}} v_{k}+\left(k^{2}-\frac{2+9 \epsilon-3 \eta}{\tau^{2}}\right) v_{k}=0
$$

Here $\epsilon$ and $\eta$ are the slow roll parameters which we assume to be small and slowly varying,

$$
\epsilon=\frac{1}{16 \pi G}\left(\frac{V_{, \varphi}}{V}\right)^{2} \ll 1, \eta=\frac{1}{24 \pi G}\left(\frac{V_{, \varphi \varphi}}{V}\right)=\frac{m_{P}^{2} m_{\varphi}^{2}}{3 V} \ll 1 .
$$

At early time, $|\tau| \gg 1 / k$ the mass term can be neglected and the field is oscillating like a free plane wave. At $1 /|\tau| \simeq k$ fluctuations are generated. 


\section{Quantum fluctuations of the inflaton IV}

The general solution of the mode equation, neglecting the time dependence of the slow role parameters, is

$$
v=(k \tau)^{1 / 2}\left(C_{1} H_{\mu}^{(1)}(k \tau)+C_{2} H_{\mu}^{(2)}(k \tau)\right), \quad \mu=3 / 2+3 \epsilon-\eta
$$

Here $H_{\mu}^{(1,2)}$ is the Hankel function of the first respectively second kind. 


\section{Quantum fluctuations of the inflaton IV}

The general solution of the mode equation, neglecting the time dependence of the slow role parameters, is

$$
v=(k \tau)^{1 / 2}\left(C_{1} H_{\mu}^{(1)}(k \tau)+C_{2} H_{\mu}^{(2)}(k \tau)\right), \quad \mu=3 / 2+3 \epsilon-\eta
$$

Here $H_{\mu}^{(1,2)}$ is the Hankel function of the first respectively second kind.

The initial condition for $v_{k}$ is determined by the requirement, that at very early times $v$ be in the vacuum state, $v_{k}(\tau)=\frac{1}{\sqrt{2 k}} \exp (-i k \tau)$. This requires that $C_{1}=0$ and determines $C_{2} \propto 1 / \sqrt{k}$. 


\section{Quantum fluctuations of the inflaton IV}

The general solution of the mode equation, neglecting the time dependence of the slow role parameters, is

$$
v=(k \tau)^{1 / 2}\left(C_{1} H_{\mu}^{(1)}(k \tau)+C_{2} H_{\mu}^{(2)}(k \tau)\right), \quad \mu=3 / 2+3 \epsilon-\eta
$$

Here $H_{\mu}^{(1,2)}$ is the Hankel function of the first respectively second kind.

The initial condition for $v_{k}$ is determined by the requirement, that at very early times $v$ be in the vacuum state, $v_{k}(\tau)=\frac{1}{\sqrt{2 k}} \exp (-i k \tau)$. This requires that $C_{1}=0$ and determines $C_{2} \propto 1 / \sqrt{k}$.

At early times, $k|\tau| \gg 1$ this describes simply a plane wave. At late times, $k|\tau| \ll 1$ it becomes a power law, $H_{\mu}^{(2)} \propto Y_{\mu} \propto X^{-\mu}$. 


\section{Quantum fluctuations of the inflaton IV}

The general solution of the mode equation, neglecting the time dependence of the slow role parameters, is

$$
v=(k \tau)^{1 / 2}\left(C_{1} H_{\mu}^{(1)}(k \tau)+C_{2} H_{\mu}^{(2)}(k \tau)\right), \quad \mu=3 / 2+3 \epsilon-\eta
$$

Here $H_{\mu}^{(1,2)}$ is the Hankel function of the first respectively second kind.

The initial condition for $v_{k}$ is determined by the requirement, that at very early times $v$ be in the vacuum state, $v_{k}(\tau)=\frac{1}{\sqrt{2 k}} \exp (-i k \tau)$. This requires that $C_{1}=0$ and determines $C_{2} \propto 1 / \sqrt{k}$.

At early times, $k|\tau| \gg 1$ this describes simply a plane wave. At late times, $k|\tau| \ll 1$ it becomes a power law, $H_{\mu}^{(2)} \propto Y_{\mu} \propto x^{-\mu}$.

What happens here is called squeezing: of the 2 solutions to the mode equation for small $x=k|\tau|$, which are $\propto x^{-\mu+1 / 2}$ and $x^{\mu+1 / 2}$, only the first survives. For cosmologically relevant modes, the suppression of the second wrt. the first mode is $x^{2 \mu} \sim 10^{-150}$ at the end of inflation. 


\section{Quantum fluctuations of the inflaton $\mathrm{V}$}

With $P_{\zeta}(k, t)=k^{3}\left\langle\left. 0|| v_{k}(t)\right|^{2} \mid 0\right\rangle /(a z)^{2}$, we obtain the power spectrum of the zeta-variable,

$$
P_{\zeta}(k, t)=\frac{2 \pi H^{2}}{5 \epsilon m_{P}^{2}}\left(\frac{k}{a H}\right)^{-6 \epsilon+2 \eta}=A_{s}\left(k / k_{*}\right)^{n_{s}-1}, \quad k /(a H) \ll 1 .
$$

The amplitude $A_{s}$ and the spectral index $n_{s}$ of the scalar spectrum are determined by the scale of inflation, $H$ and the slow roll parameters, $n_{s}-1=-6 \epsilon+2 \eta$. 


\section{Quantum fluctuations of the inflaton V}

With $P_{\zeta}(k, t)=k^{3}\left\langle\left. 0|| v_{k}(t)\right|^{2} \mid 0\right\rangle /(a z)^{2}$, we obtain the power spectrum of the zeta-variable,

$$
P_{\zeta}(k, t)=\frac{2 \pi H^{2}}{5 \epsilon m_{P}^{2}}\left(\frac{k}{a H}\right)^{-6 \epsilon+2 \eta}=A_{s}\left(k / k_{*}\right)^{n_{s}-1}, \quad k /(a H) \ll 1 .
$$

The amplitude $A_{s}$ and the spectral index $n_{s}$ of the scalar spectrum are determined by the scale of inflation, $H$ and the slow roll parameters, $n_{s}-1=-6 \epsilon+2 \eta$.

After inflation, the curvature fluctuation $\zeta$ is simply related to the metric fluctuation by $P_{\psi}=\frac{4}{9} P_{\zeta}$ in a radiation dominated Universe $(k \tau \ll 1)$ and $P_{\Psi}=\frac{9}{25} P_{\zeta}$ in a matter dominated Universe. 


\section{Quantum fluctuations of the inflaton: resume}

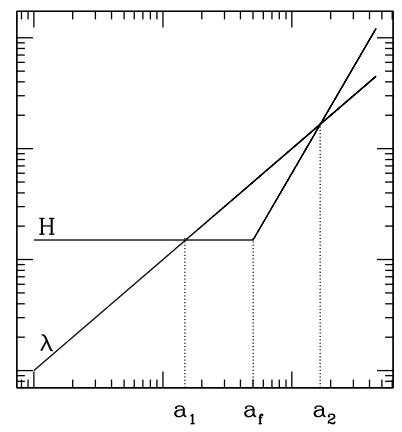

The physical wavelength $\lambda$ (inverse of frequency) of a mode is growing faster than the Hubble scale $\mathrm{H}^{-1}$ during inflation. After inflation, in the radiation phase, $\lambda \propto a \propto \sqrt{t}$ while $H^{-1} \propto t$, the Hubble scale catches up. 


\section{Quantum fluctuations of the inflaton: resume}

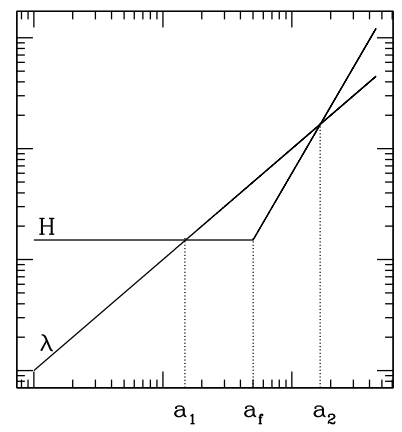

The physical wavelength $\lambda$ (inverse of frequency) of a mode is growing faster than the Hubble scale $\mathrm{H}^{-1}$ during inflation. After inflation, in the radiation phase, $\lambda \propto a \propto \sqrt{t}$ while $H^{-1} \propto t$, the Hubble scale catches up.

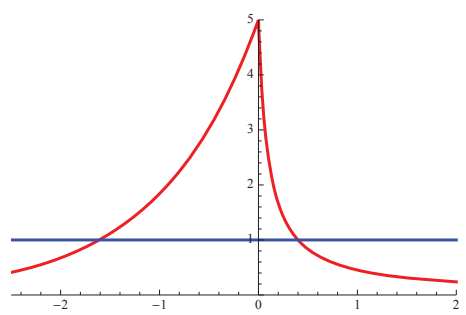

Even simpler, we can regard it as a quantum mechanical scattering process with incoming plane wave and potential $\ddot{z} /\left(a^{2} z\right)$.

Note: the 'quantum aspect' of the treatment is that we know the initial conditions, the quantum vacuum. As usual, the evolution equation for the amplitudes of the quantum modes is the classical equation of motion. 


\section{Quantum fluctuations of the graviton I}

The freely propagating mode of gravitation itself is what we call the graviton. It is given by the transverse traceless perturbations of the metric,

$$
d s^{2}=a^{2}\left(-d \tau^{2}+\left(\delta_{i j}+2 H_{i j}\right) d x^{i} d x^{j}\right), H_{j}^{i}=0, H_{, j}^{i j}=0
$$




\section{Quantum fluctuations of the graviton I}

The freely propagating mode of gravitation itself is what we call the graviton. It is given by the transverse traceless perturbations of the metric,

$$
d s^{2}=a^{2}\left(-d \tau^{2}+\left(\delta_{i j}+2 H_{i j}\right) d x^{i} d x^{j}\right), H_{j}^{i}=0, H_{, j}^{i j}=0
$$

To second order, the perturbed action is

$$
\begin{gathered}
S+\delta S=-\frac{m_{p}^{2}}{16 \pi} \int d^{4} x \sqrt{-(g+\delta g)}(R+\delta R) \\
\delta S=\delta S_{+}+\delta S_{-}, \quad \delta S_{ \pm}=\frac{1}{2} \int d^{4} x\left[\left(\partial_{\tau} h_{ \pm}\right)^{2}-\left(\nabla h_{ \pm}\right)^{2}+\frac{\ddot{a}}{a} h_{ \pm}^{2}\right]
\end{gathered}
$$

where $H_{i j}=\frac{\sqrt{8 \pi}}{m_{p}}\left(h_{+} e_{i j}^{(+)}+h_{-} e_{i j}^{(-)}\right)$. 


\section{Quantum fluctuations of the graviton I}

The freely propagating mode of gravitation itself is what we call the graviton. It is given by the transverse traceless perturbations of the metric,

$$
d s^{2}=a^{2}\left(-d \tau^{2}+\left(\delta_{i j}+2 H_{i j}\right) d x^{i} d x^{j}\right), H_{j}^{i}=0, H^{i j},=0
$$

To second order, the perturbed action is

$$
\begin{gathered}
S+\delta S=-\frac{m_{p}^{2}}{16 \pi} \int d^{4} x \sqrt{-(g+\delta g)}(R+\delta R) \\
\delta S=\delta S_{+}+\delta S_{-}, \quad \delta S_{ \pm}=\frac{1}{2} \int d^{4} x\left[\left(\partial_{\tau} h_{ \pm}\right)^{2}-\left(\nabla h_{ \pm}\right)^{2}+\frac{\ddot{a}}{a} h_{ \pm}^{2}\right]
\end{gathered}
$$

where $H_{i j}=\frac{\sqrt{8 \pi}}{m_{p}}\left(h_{+} e_{i j}^{(+)}+h_{-} e_{i j}^{(-)}\right)$.

The fields $h_{ \pm}$satisfy the same mode equations as $v$ with $z$ replaced by the scale factor $a$.

$$
\frac{d^{2}}{d \tau^{2}} h_{k}+\left(k^{2}-\frac{\ddot{a}}{a}\right) h_{k}=0
$$




\section{Quantum fluctuations of the graviton II}

Correspondingly they have the same solutions, just with the Hankel function index $\mu$ replaced by $\nu=3 / 2+\epsilon$,

$$
h=B_{2}|\tau|^{1 / 2} H_{\nu}^{(2)}(k \tau) .
$$

With this we obtain the tensor spectrum on super Hubble scales, $k / a \ll H$,

$$
k^{3} P_{T}=32 \pi \frac{H^{2}}{m_{P}^{2}}\left(\frac{k}{a H}\right)^{-2 \epsilon}=A_{t}\left(k / k_{*}\right)^{-2 \epsilon}
$$

Neglecting the running with $\epsilon \ll 1$, the amplitude of tensor fluctuations directly determines the scale of inflation,

$$
A_{t}=32 \pi\left(H / m_{P}\right)^{2}=\frac{4}{3} \frac{V}{m_{P}^{4}}
$$




\section{Quantum fluctuations of the graviton II}

Correspondingly they have the same solutions, just with the Hankel function index $\mu$ replaced by $\nu=3 / 2+\epsilon$,

$$
h=B_{2}|\tau|^{1 / 2} H_{\nu}^{(2)}(k \tau) .
$$

With this we obtain the tensor spectrum on super Hubble scales, $k / a \ll H$,

$$
k^{3} P_{T}=32 \pi \frac{H^{2}}{m_{P}^{2}}\left(\frac{k}{a H}\right)^{-2 \epsilon}=A_{t}\left(k / k_{*}\right)^{-2 \epsilon}
$$

Neglecting the running with $\epsilon \ll 1$, the amplitude of tensor fluctuations directly determines the scale of inflation,

$$
A_{t}=32 \pi\left(H / m_{P}\right)^{2}=\frac{4}{3} \frac{V}{m_{P}^{4}}
$$

Comparing it to the amplitude os scalar perturbations one obtains the consistency relation for slow roll inflation

$$
r=P_{T} / P_{\zeta}=16 \epsilon=-8 n_{t}
$$




\section{Quantum fluctuations of the graviton III}

In the above calculation the perturbation of the metric has been treated as a quantum field. The detection of such a background, e.g. in the CMB would therefore provide direct evidence of quantum gravity! 


\section{Quantum fluctuations of the graviton III}

In the above calculation the perturbation of the metric has been treated as a quantum field. The detection of such a background, e.g. in the CMB would therefore provide direct evidence of quantum gravity!

In the CMB temperature anisotropies it is difficult to separate a gravitational wave contribution for the scalar contribution. 


\section{Quantum fluctuations of the graviton III}

In the above calculation the perturbation of the metric has been treated as a quantum field. The detection of such a background, e.g. in the CMB would therefore provide direct evidence of quantum gravity!

In the CMB temperature anisotropies it is difficult to separate a gravitational wave contribution for the scalar contribution.

Parameter estimation via Markov Chain Monte Carlo (MCMC) methods have so far given $r<0$.3. 


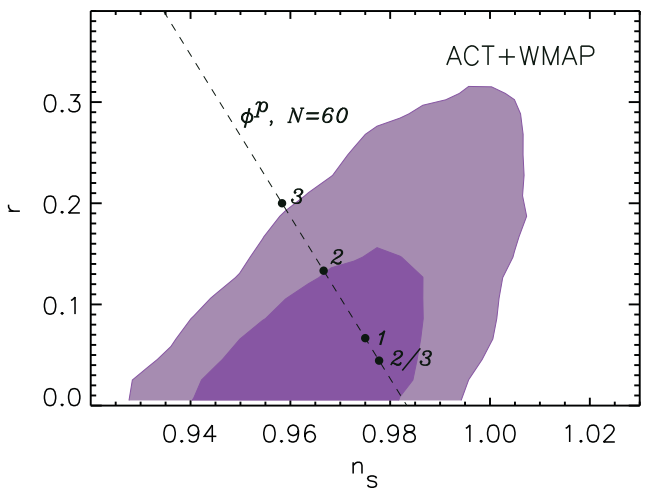

2D marginalized limits $(68 \%$ and $95 \% \mathrm{CL}$ ) for the tensor-to-scalar ratio $r$, and the scalar spectral index $n_{s}$, for ACT+WMAP data. By measuring the $\ell>1000$ spectrum, the longer lever arm from ACT data further breaks the $n_{s}-r$ degeneracy, giving a marginalized limit $r<0.25$ (95\% CL) from the CMB alone. The predicted values for a chaotic inflationary model with inflaton potential $V(\phi) \propto \phi^{p}$ with 60 e-folds are shown for $p=3,2,1,2 / 3 ; p>3$ is disfavored at $>95 \% \mathrm{CL}$.

J. Dunkley et al. 2010. 
- Inflation ends by converting the inflaton energy density into massless particles which are in thermal equilibrium. By this process a hot radiation dominated universe is obtained. This is the modern cosmology version of the 'hot big bang'. 
- Inflation ends by converting the inflaton energy density into massless particles which are in thermal equilibrium. By this process a hot radiation dominated universe is obtained. This is the modern cosmology version of the 'hot big bang'.

- The details of the pre-heating and reheating process are complicated, model-dependent and not fully understood. 
- Inflation ends by converting the inflaton energy density into massless particles which are in thermal equilibrium. By this process a hot radiation dominated universe is obtained. This is the modern cosmology version of the 'hot big bang'.

- The details of the pre-heating and reheating process are complicated, model-dependent and not fully understood.

- After inflation, the results we have obtained are valid on super Hubble scales, $k / a_{*} \ll H_{*}$, i.e scales which are larger than the Hubble scale at the end of inflation. 
- Inflation ends by converting the inflaton energy density into massless particles which are in thermal equilibrium. By this process a hot radiation dominated universe is obtained. This is the modern cosmology version of the 'hot big bang'.

- The details of the pre-heating and reheating process are complicated, model-dependent and not fully understood.

- After inflation, the results we have obtained are valid on super Hubble scales, $k / a_{*} \ll H_{*}$, i.e scales which are larger than the Hubble scale at the end of inflation.

- In a decelerating universe, both $\zeta$ and $H_{i j}$ are constant on large scales, $k / a(t) \ll H(t)$. 


\section{The post-inflationary Universe}

Once the fluctuations enter the horizon, gravitational waves oscillate like $\cos (k \tau)$ and decay like $1 / a$. 


\section{The post-inflationary Universe}

Once the fluctuations enter the horizon, gravitational waves oscillate like $\cos (k \tau)$ and decay like $1 / a$.

Scalar perturbations depend on the matter equation of state:

- During radiation domination they also oscillate like $\cos \left(c_{s} k \tau\right)$ and decay like $1 / a^{2}$, the energy density fluctuations oscillate with constant amplitude.

- During matter domination $\zeta$ remains constant, the density fluctuation grows like $a$. 
Once the fluctuations enter the horizon, gravitational waves oscillate like $\cos (k \tau)$ and decay like $1 / a$.

Scalar perturbations depend on the matter equation of state:

- During radiation domination they also oscillate like $\cos \left(c_{s} k \tau\right)$ and decay like $1 / a^{2}$, the energy density fluctuations oscillate with constant amplitude.

- During matter domination $\zeta$ remains constant, the density fluctuation grows like $a$.

- This behavior in imprinted in the CMB spectrum via the linear transfer functions

$$
T_{(S, T)}(\ell, k) \propto \min \left\{1,\left(k \tau_{\text {eq }}\right)^{-4}\right\} \cos ^{2}\left(c_{s} k \tau_{\mathrm{dec}}\right) k^{3} j_{\ell}^{2}\left(k \tau_{\mathrm{dec}}\right) \cdots
$$

which depends on cosmological parameters. 
Once the fluctuations enter the horizon, gravitational waves oscillate like $\cos (k \tau)$ and decay like $1 / a$.

Scalar perturbations depend on the matter equation of state:

- During radiation domination they also oscillate like $\cos \left(c_{s} k \tau\right)$ and decay like $1 / a^{2}$, the energy density fluctuations oscillate with constant amplitude.

- During matter domination $\zeta$ remains constant, the density fluctuation grows like $a$.

- This behavior in imprinted in the CMB spectrum via the linear transfer functions

$$
T_{(S, T)}(\ell, k) \propto \min \left\{1,\left(k \tau_{\text {eq }}\right)^{-4}\right\} \cos ^{2}\left(c_{s} k \tau_{\mathrm{dec}}\right) k^{3} j_{\ell}^{2}\left(k \tau_{\mathrm{dec}}\right) \cdots
$$

which depends on cosmological parameters.

- Non-inflationary perturbations can only be generated once a given scale has entered the horizon. They typically lead to

$$
T_{(S, T)}(\ell, k) \propto \min \left\{1,\left(k \tau_{e q}\right)^{-4}\right\} \cos ^{2}\left(c_{s} k \tau_{\mathrm{dec}}+\phi\right) k^{3} j_{\ell}^{2}\left(k \tau_{\mathrm{dec}}\right) \cdots
$$

with random phase $\phi$. 


\section{Topological defects}

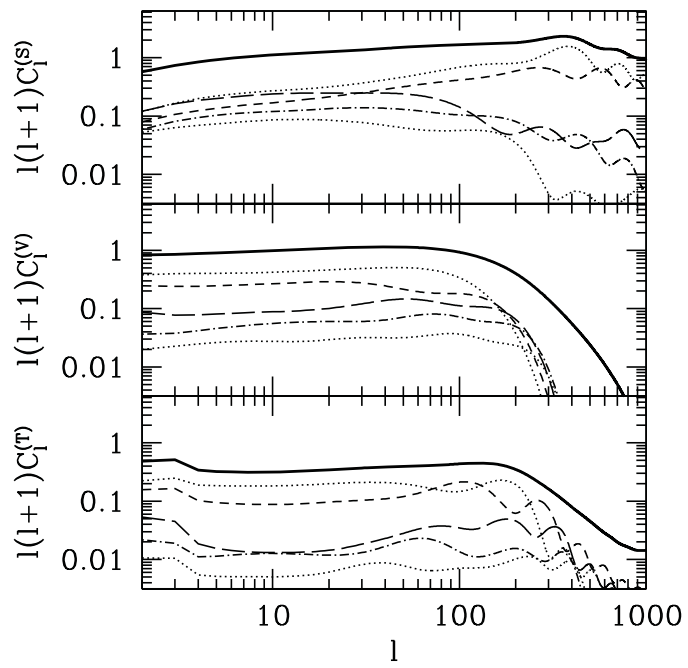

CMB anisotropies from global texture (Bevis et al. 2004). 


\section{Bardeen potential}

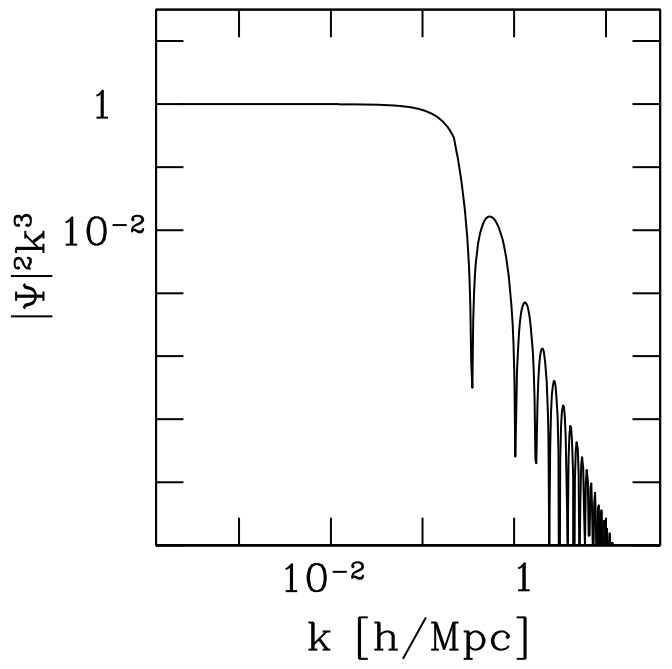

Sketch of the Bardeen potential (proportional to $\zeta$ ) at late times. (from RD, '08) 
CMB anisotropies from scalar and tensor fluctuations

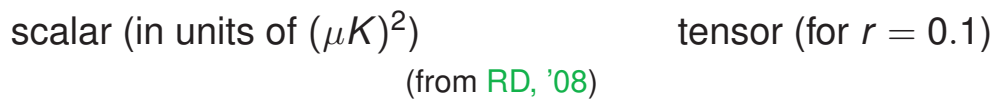
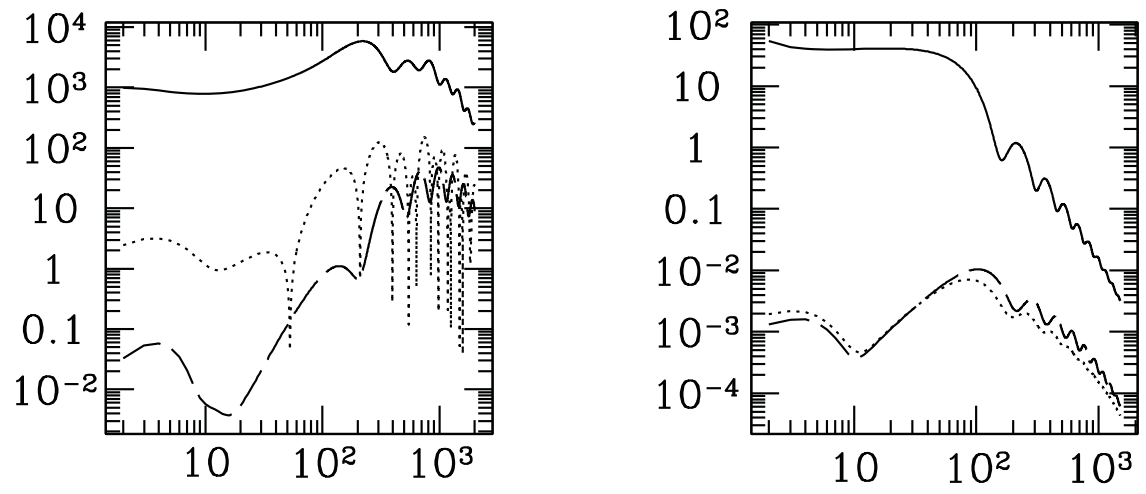


\section{CMB Polarization}

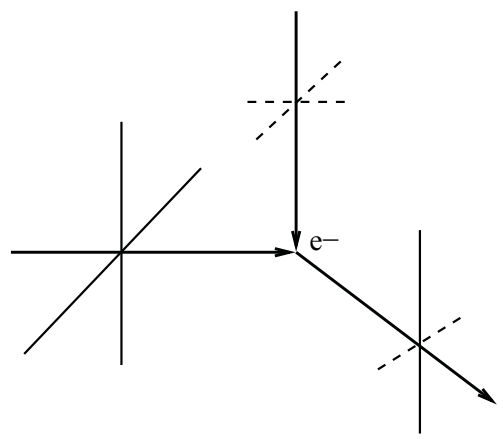

$$
\begin{aligned}
E_{\|}^{(c)} & =\sqrt{\frac{3}{8 \pi} n_{e} \sigma_{T}} \cos \theta E_{\|} \\
E_{\perp}^{(c)} & =\sqrt{\frac{3}{8 \pi} n_{e} \sigma_{T}} E_{\perp}
\end{aligned}
$$

(from RD, '08)

The Thomson cross section depends on polarization. A quadrupole anisotropy on the last scattering surface induces polarisation. 


\section{CMB polarization from scalar and tensor fluctuations}

\section{E-type polarisation pattern B-type polarisation pattern}
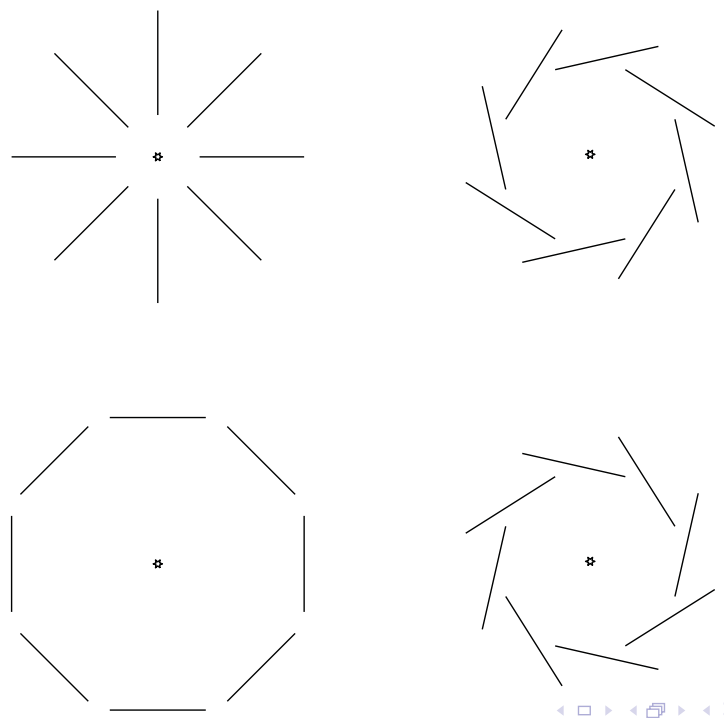


\section{E- and B-polarization from scalar and tensor fluctuations I}
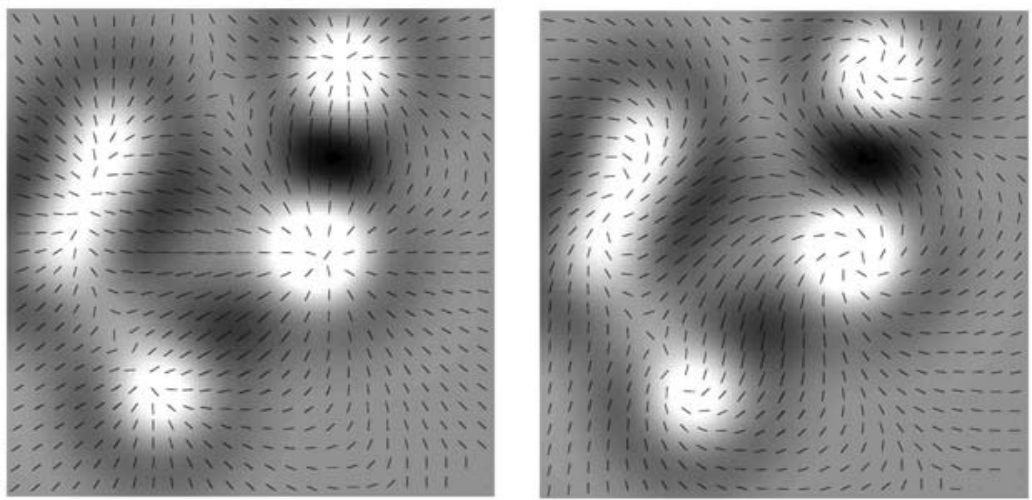

(from RD, '08)

A E-polarization pattern (left) is compared with B-polarization. The function $\tilde{\mathcal{E}}=\tilde{\mathcal{B}}$ is indicated in grey scale, and the polarization directions are drawn. E-polarization is tangential along the dark negative regions while it is radial from the white white positive regions. The B-polarization pattern can be obtained by rotating the polarization directions by $45^{\circ}$. 
CMB polarisation from scalar and tensor fluctuations III

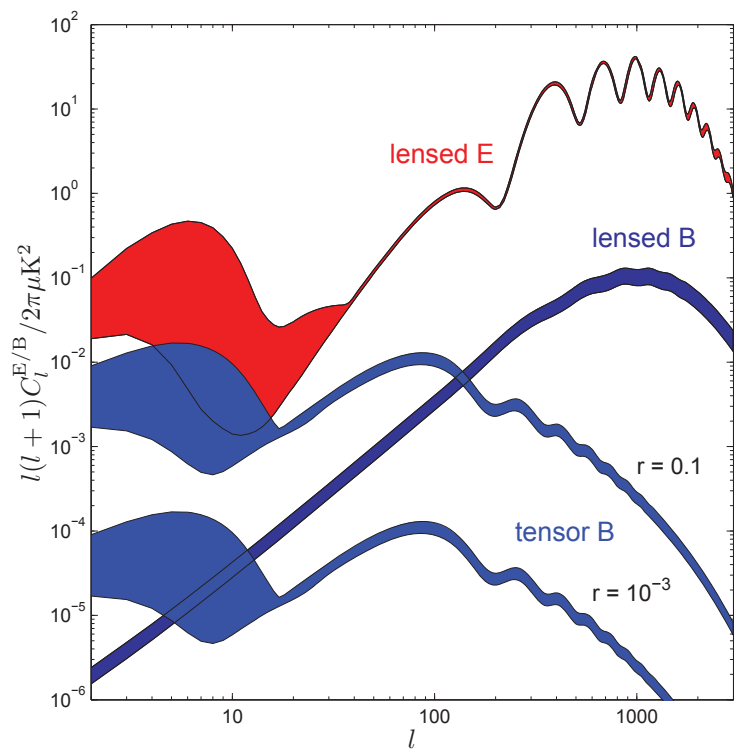

(from Challinor \& Lewis '06) 


\section{Post-inflationary fluctuations}

In this discussion I have neglected the fact that the $\zeta$ and $H$ which we compute during inflation are quantum fields and those which we measure are classical fluctuations. 


\section{Post-inflationary fluctuations}

In this discussion I have neglected the fact that the $\zeta$ and $H$ which we compute during inflation are quantum fields and those which we measure are classical fluctuations.

One can show that in usual circumstances this is OK. The quantum fluctuations do decohere and become classical fluctuations with the same spectrum (see eg. Kiefer et al., 1998). It is easy to see that the occupation numbers are large. But the state is strongly squeezed (only the growing mode survives). 


\section{Post-inflationary fluctuations}

In this discussion I have neglected the fact that the $\zeta$ and $H$ which we compute during inflation are quantum fields and those which we measure are classical fluctuations.

One can show that in usual circumstances this is OK. The quantum fluctuations do decohere and become classical fluctuations with the same spectrum (see eg. Kiefer et al., 1998). It is easy to see that the occupation numbers are large. But the state is strongly squeezed (only the growing mode survives).

In this situation a squeezed state can be approximated by a classical random field with random amplitude and fixed phase. The quantum to classical transition is induced not by the usual decoherence due to interactions with an environment, but by the decay of the quantum correlation between the growing and the decaying mode "decoherence without decoherence". 


\section{Post-inflationary fluctuations}

In this discussion I have neglected the fact that the $\zeta$ and $H$ which we compute during inflation are quantum fields and those which we measure are classical fluctuations.

One can show that in usual circumstances this is OK. The quantum fluctuations do decohere and become classical fluctuations with the same spectrum (see eg. Kiefer et al., 1998). It is easy to see that the occupation numbers are large. But the state is strongly squeezed (only the growing mode survives).

In this situation a squeezed state can be approximated by a classical random field with random amplitude and fixed phase. The quantum to classical transition is induced not by the usual decoherence due to interactions with an environment, but by the decay of the quantum correlation between the growing and the decaying mode "decoherence without decoherence".

The fact that the phase of the fluctuations (of a given wave number) is fixed is actually very important. It is responsible the the acoustic pics in the CMB anisotropy spectrum. 


\section{Post-inflationary fluctuations}

Since they originate from quantum fluctuations of a nearly free field we expect the fluctuations to be nearly Gaussian. One can show that for single field slow roll inflation, non-Gaussianities are small, of the order of the slow roll parameters, i.e. the bi-spectrum defined by

$$
k_{1}^{3} k_{2}^{3}\left\langle\zeta\left(\mathbf{k}_{1}\right) \zeta\left(\mathbf{k}_{2}\right) \zeta\left(\mathbf{k}_{2}\right)\right\rangle \equiv(2 \pi)^{6} \delta\left(\mathbf{k}_{1}+\mathbf{k}_{2}+\mathbf{k}_{3}\right) B\left(k_{1}, k_{2}, k_{3}\right)
$$

is $k^{6} B=f_{n l}\left(k^{3} P\right)^{2} \simeq \epsilon\left(k^{3} P\right)^{2}$, (an $f_{n l} \sim 1$ is expected from the non-linearity of the fluctuations).

There are many models of inflation, some of them not slow-roll, which can lead to a fluctuation spectrum compatible with observations. Improving limits on non-Gaussianity and on B-polarisation from gravitational waves are crucial to distinguish them. 


\section{Planck satellite}

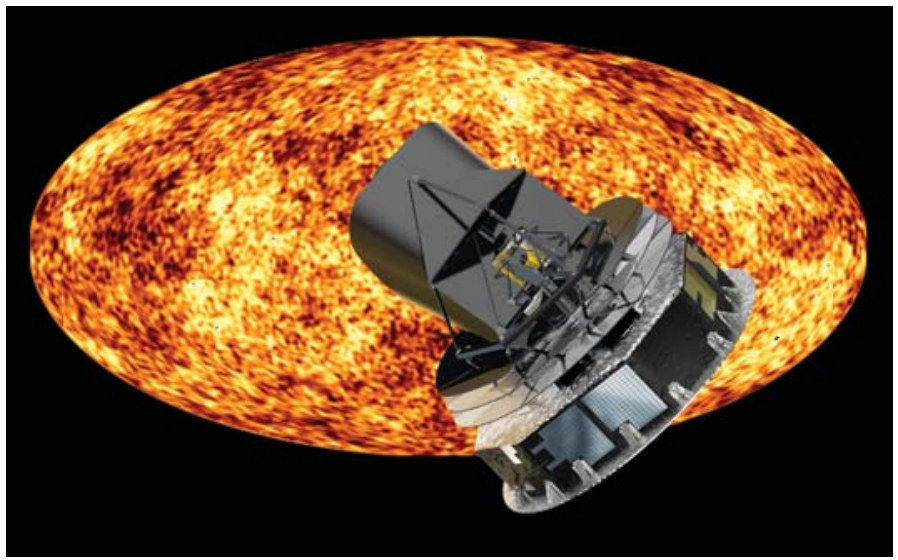

The Planck satellite of ESA launched in 2009 has observed the microwave sky in more than 20 frequencies with a resolution of a few arc minutes for about 2.5 years ( 5 full sky coverages). Results are expected soon. Can detect $r \geq 0.05, \quad t_{n l} \gtrsim$ a few. 


\section{Post-inflationary fluctuations}

Since they originate from quantum fluctuations of a free field we expect the fluctuations to be Gaussian. One can show that for single field slow roll inflation, non-Gaussianities are small, of the order of the slow roll parameters, i.e. the bi-spectrum defined by

$$
k_{1}^{3} k_{2}^{3}\left\langle\zeta\left(\mathbf{k}_{1}\right) \zeta\left(\mathbf{k}_{2}\right) \zeta\left(\mathbf{k}_{2}\right)\right\rangle \equiv(2 \pi)^{6} \delta\left(\mathbf{k}_{1}+\mathbf{k}_{2}+\mathbf{k}_{3}\right) B\left(k_{1}, k_{2}, k_{3}\right)
$$

is $k^{6} B=f_{n l}\left(k^{3} P\right)^{2} \simeq \epsilon\left(k^{3} P\right)^{2}$, (an $f_{n l} \sim 1$ is expected from the non-linearity of the fluctuations).

There are many models of inflation, some of them not slow-roll, which can lead to a fluctuation spectrum compatible with observations. Improving limits on non-Gaussianity and on B-polarisation from gravitational waves are crucial to distinguish them.

In addition better observations and understanding of the more complicated matter distribution is needed. This gives us 3-dimensional information while the CMB is only 2-dimensional. 


\section{Euclid an ESA M-class mission approved for launch in 2019}

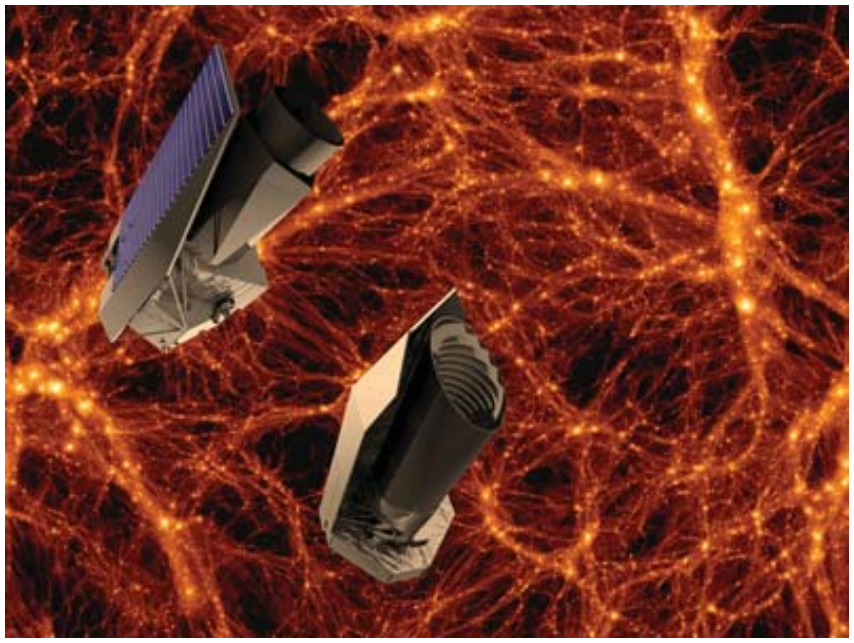




\section{Conclusions}

- According to our present understanding of cosmology, the largest fluctuations in the Universe, visible in the CMB and in the large scale distribution of galaxies and clusters have their origin in tiny quantum fluctuations which have subsequently expanded to cosmological scales! 


\section{Conclusions}

- According to our present understanding of cosmology, the largest fluctuations in the Universe, visible in the CMB and in the large scale distribution of galaxies and clusters have their origin in tiny quantum fluctuations which have subsequently expanded to cosmological scales!

- Even if the details of inflation and especially the origin of the inflaton, are presently still unclear, this seems to me the most stunning success of combining general relativity and quantum field theory. Especially, the CMB spectrum might be our first evidence of 'particle' (quantum mode) generation in a classical external field! 


\section{Conclusions}

- According to our present understanding of cosmology, the largest fluctuations in the Universe, visible in the CMB and in the large scale distribution of galaxies and clusters have their origin in tiny quantum fluctuations which have subsequently expanded to cosmological scales!

- Even if the details of inflation and especially the origin of the inflaton, are presently still unclear, this seems to me the most stunning success of combining general relativity and quantum field theory. Especially, the CMB spectrum might be our first evidence of 'particle' (quantum mode) generation in a classical external field!

- If we can detect tensor fluctuations in the CMB this would be direct evidence of the quantum nature of spacetime. 
RD \& M. Ruser, arXiv:0704.0756, PRL 99, 071601 (2007)

M. Ruser \& RD, arXiv:0704.0790, PRD 76, 104014 (2007)

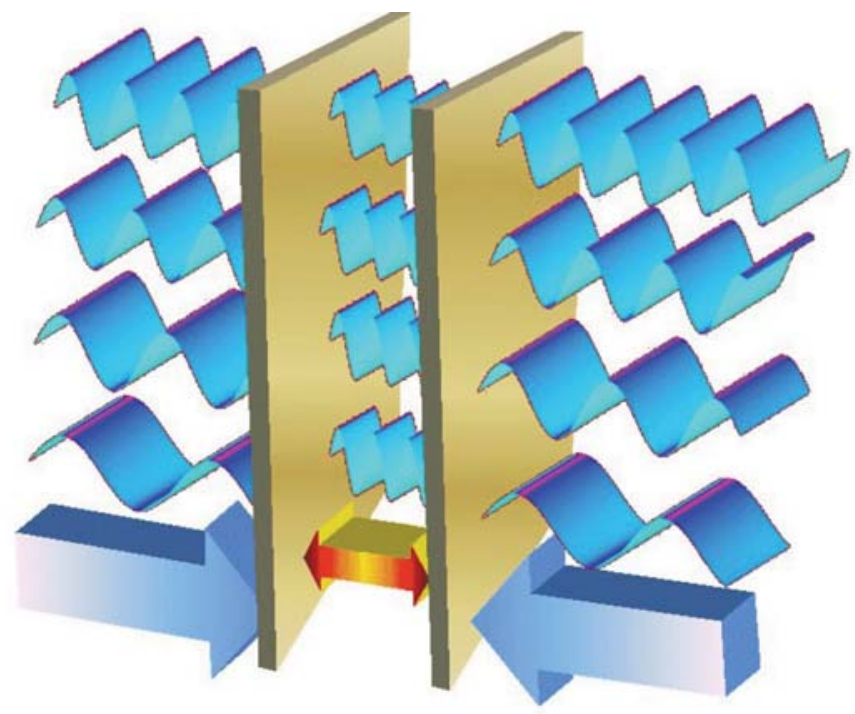




\section{Braneworlds}

- String theory, the most serious candidate for a quantum theory of gravity, motivates the existence of braneworlds to which standard model particles are confined. 


\section{Braneworlds}

- String theory, the most serious candidate for a quantum theory of gravity, motivates the existence of braneworlds to which standard model particles are confined.

- In (the minimal version of) these models only the graviton propagates in the full spacetime, the bulk. 


\section{Braneworlds}

- String theory, the most serious candidate for a quantum theory of gravity, motivates the existence of braneworlds to which standard model particles are confined.

- In (the minimal version of) these models only the graviton propagates in the full spacetime, the bulk.

- The expansion of the universe can be modeled by the motion of the brane which represents our universe through a warped bulk. 


\section{Braneworlds}

- String theory, the most serious candidate for a quantum theory of gravity, motivates the existence of braneworlds to which standard model particles are confined.

- In (the minimal version of) these models only the graviton propagates in the full spacetime, the bulk.

- The expansion of the universe can be modeled by the motion of the brane which represents our universe through a warped bulk.

- Here we consider the RS model (Randall \& Sundrum, 99) : a 4d brane moving through a $5 d$ AdS spacetime. 


\section{Braneworlds}

- String theory, the most serious candidate for a quantum theory of gravity, motivates the existence of braneworlds to which standard model particles are confined.

- In (the minimal version of) these models only the graviton propagates in the full spacetime, the bulk.

- The expansion of the universe can be modeled by the motion of the brane which represents our universe through a warped bulk.

- Here we consider the RS model (Randall \& Sundrum, 99) : a 4d brane moving through a $5 d$ AdS spacetime.

- Like a moving metal plate can lead to the generation of photons by the dynamical Casimir effect, a moving brane can lead to the generation of gravitons. 


\section{RS Braneworld}

The $5 \mathrm{~d}$ AdS metric in conformal coordinates is

$$
d s^{2}=\left(\frac{L}{y}\right)^{2}\left[\eta_{\mu \nu} d x^{\mu} d x^{\nu}+d y^{2}\right]
$$

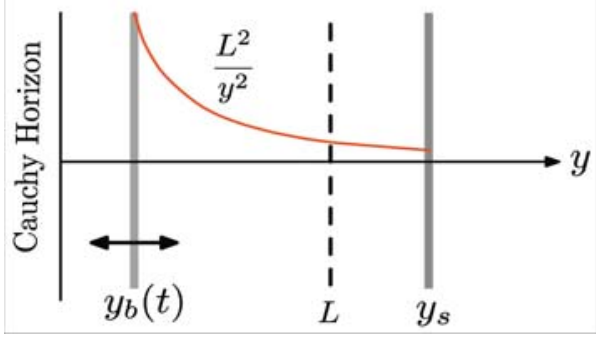

The Einstein equations, $G_{a b}=-\wedge g_{a b}$ give $\Lambda=-6 / L^{2}$.

$M_{5}^{3}=M_{4}^{2} / L$. For $L \sim 0.1 \mathrm{~mm} M_{4}=10^{19} \mathrm{GeV}$ yields $M_{5} \simeq 10^{5} \mathrm{TeV}$. 


\section{The Dynamical Casimir effect in Braneworlds}

4d metric on the brane: $d s^{2}=\left(L / y_{b}(t)\right)^{2}\left[-d \eta^{2}+\delta_{i j} d x^{i} d x^{j}\right]$

$a=L / y_{b}, d \eta=\left(1-v^{2}\right)^{1 / 2} d t=\gamma^{-1} d t, v=d y_{b} / d t$

Junction condition (at low energy):

$$
18 H^{2}=\kappa_{5}^{2} \lambda \rho=6 \kappa_{4} \rho \quad\left(8 \pi G_{4}=\right) \kappa_{4}=\kappa_{5}^{2} \lambda / 6
$$

RS fine tuning condition:

$$
\kappa_{5}^{2} \lambda^{2}=36 / L^{2} . \quad \text { With } \kappa_{5}=L_{s}^{3} \text { and } \kappa_{4}=L_{P}^{2}
$$

this yields:

$$
\frac{L}{L_{s}}=\left(\frac{L_{s}}{L_{P}}\right)^{2}
$$




\section{The Dynamical Casimir effect in Braneworlds}

One can now compute exactly like for the ordinary dynamical Casimir effect, the generation of $4 \mathrm{~d}$ gravitons due to the motion of the moving brane (our Universe).

$$
d s^{2}=\left(\frac{L}{y}\right)^{2}\left[-d \tau^{2}+\left(\delta_{i j}+2 h_{i j}\right) d x^{i} d x^{j}+d y^{2}\right]
$$




\section{The Dynamical Casimir effect in Braneworlds}

One can now compute exactly like for the ordinary dynamical Casimir effect, the generation of $4 \mathrm{~d}$ gravitons due to the motion of the moving brane (our Universe).

$$
\begin{gathered}
d s^{2}=\left(\frac{L}{y}\right)^{2}\left[-d \tau^{2}+\left(\delta_{i j}+2 h_{i j}\right) d x^{i} d x^{j}+d y^{2}\right] \\
\left(\partial_{\tau}^{2}+k^{2}-\partial_{y}^{2}-\frac{3}{y} \partial_{y}\right) h_{k}(\tau, y)=0
\end{gathered}
$$

bounadary conditions:

$$
\left.\partial_{y} h_{k}(\tau, y)\right|_{y_{b}}=\left.\partial_{y} h_{k}(\tau, y)\right|_{y_{s}}=0
$$




\section{The Dynamical Casimir effect in Braneworlds}

One can now compute exactly like for the ordinary dynamical Casimir effect, the generation of $4 \mathrm{~d}$ gravitons due to the motion of the moving brane (our Universe).

$$
\begin{gathered}
d s^{2}=\left(\frac{L}{y}\right)^{2}\left[-d \tau^{2}+\left(\delta_{i j}+2 h_{i j}\right) d x^{i} d x^{j}+d y^{2}\right] \\
\left(\partial_{\tau}^{2}+k^{2}-\partial_{y}^{2}-\frac{3}{y} \partial_{y}\right) h_{k}(\tau, y)=0
\end{gathered}
$$

bounadary conditions:

$$
\left.\partial_{y} h_{k}(\tau, y)\right|_{y_{b}}=\left.\partial_{y} h_{k}(\tau, y)\right|_{y_{s}}=0
$$

The spatial part of this eqn. forms a Sturm Liouville problem with a complete orthonormal set of solutions, the instantaneous mode functions $\phi_{\alpha}(k, \tau, y)$ which can be determined in terms of Bessel functions.

$$
h_{k}(\tau, y)=\sum_{\alpha} q_{\alpha}(\tau) \phi_{\alpha}(k, \tau, y) \quad \alpha \in \mathbb{N}_{0}, .
$$




\section{The Dynamical Casimir effect in Braneworlds}

One can now compute exactly like for the ordinary dynamical Casimir effect, the generation of $4 \mathrm{~d}$ gravitons due to the motion of the moving brane (our Universe).

$$
\begin{gathered}
d s^{2}=\left(\frac{L}{y}\right)^{2}\left[-d \tau^{2}+\left(\delta_{i j}+2 h_{i j}\right) d x^{i} d x^{j}+d y^{2}\right] \\
\left(\partial_{\tau}^{2}+k^{2}-\partial_{y}^{2}-\frac{3}{y} \partial_{y}\right) h_{k}(\tau, y)=0
\end{gathered}
$$

bounadary conditions:

$$
\left.\partial_{y} h_{k}(\tau, y)\right|_{y_{b}}=\left.\partial_{y} h_{k}(\tau, y)\right|_{y_{s}}=0
$$

The spatial part of this eqn. forms a Sturm Liouville problem with a complete orthonormal set of solutions, the instantaneous mode functions $\phi_{\alpha}(k, \tau, y)$ which can be determined in terms of Bessel functions.

$$
h_{k}(\tau, y)=\sum_{\alpha} q_{\alpha}(\tau) \phi_{\alpha}(k, \tau, y) \quad \alpha \in \mathbb{N}_{0}, .
$$

$\alpha=0$ corresponds to the usual massless $4 \mathrm{~d}$ graviton while $\alpha=j \in \mathbb{N}$ are massive Kaluza-Klein modes. Their mass is given by their momentum in $y$-direction. 


\section{The Dynamical Casimir effect in Braneworlds}

The time dependent boundary conditions lead to coupled second order differential eons. for $q_{\alpha}(t)$ which determines the particle production. The produced massive gravitons, $\omega_{\alpha}^{2}=k^{2}+m_{\alpha}^{2}$ have a time dependent mass, $m_{j} \simeq j \pi /\left(y_{s}-y_{b}\right)$ which is redshifted with the expansion of the Universe. 
The time dependent boundary conditions lead to coupled second order differential eons. for $q_{\alpha}(t)$ which determines the particle production. The produced massive gravitons, $\omega_{\alpha}^{2}=k^{2}+m_{\alpha}^{2}$ have a time dependent mass, $m_{j} \simeq j \pi /\left(y_{s}-y_{b}\right)$ which is redshifted with the expansion of the Universe.

The energy density of the massless graviton goes like

$$
\rho_{0} \propto\left|h^{\prime}\right|^{2} / a^{2} \propto k^{2}\left|\phi_{0}\right|^{2} / a^{2} \propto a^{-4} \quad \text { (radiation) }
$$

While the density of the massive modes goes like

$$
\rho_{n} \propto\left|h^{\prime}\right|^{2} / a^{2} \propto \omega^{2}\left|\phi_{n}\right|^{2} / a^{2} \propto a^{-6} \quad \text { (stiff matter) }
$$


The Dynamical Casimir effect in Braneworlds

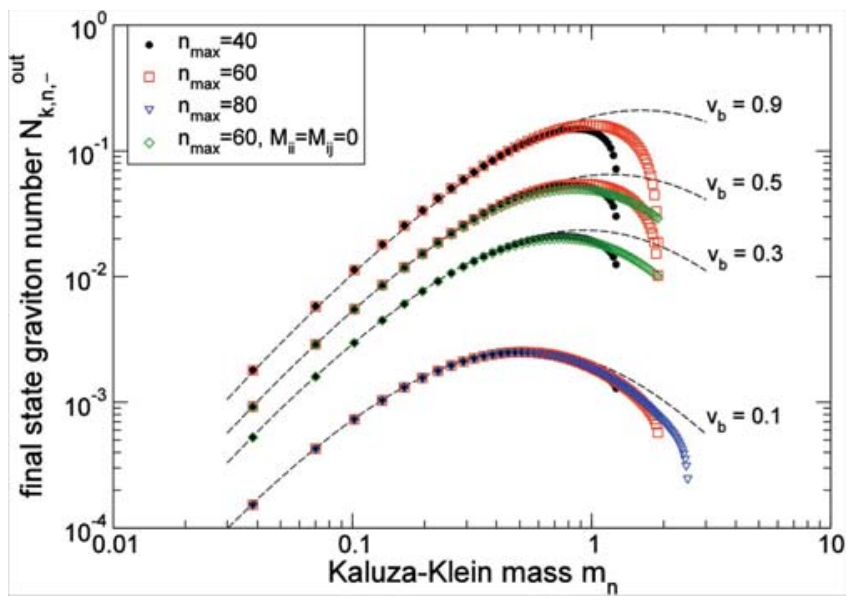

$$
\text { for } y_{s}=100
$$

$$
\Omega_{h 0} \simeq v_{b} \Omega_{\mathrm{rad}} \quad \frac{\Omega_{h K K}\left(\tau_{b}\right)}{\Omega_{\mathrm{rad}}\left(\tau_{b}\right)} \simeq 100 v_{b}^{3} \frac{L}{y_{s}}\left(\frac{L}{L_{s}}\right)^{2} \sim v_{b}^{3} \frac{L}{y_{s}} 10^{42}
$$


- Braneworld cosmology where expansion is mimicked by a brane moving through a warped higher dimensional spacetime leads to particle creation via the dynamical Casimir effect for bulk modes. 
- Braneworld cosmology where expansion is mimicked by a brane moving through a warped higher dimensional spacetime leads to particle creation via the dynamical Casimir effect for bulk modes.

- KK gravitons scale like stiff matter, $1 / a^{6}$, and can therefore not represent dark matter. 
- Braneworld cosmology where expansion is mimicked by a brane moving through a warped higher dimensional spacetime leads to particle creation via the dynamical Casimir effect for bulk modes.

- KK gravitons scale like stiff matter, $1 / a^{6}$, and can therefore not represent dark matter.

- In an ekpyrotic type scenario with an AdS5 bulk, the nucleosynthesis bound on gravitational waves requires $v_{b}<0.2$. 
- Braneworld cosmology where expansion is mimicked by a brane moving through a warped higher dimensional spacetime leads to particle creation via the dynamical Casimir effect for bulk modes.

- KK gravitons scale like stiff matter, $1 / a^{6}$, and can therefore not represent dark matter.

- In an ekpyrotic type scenario with an AdS5 bulk, the nucleosynthesis bound on gravitational waves requires $v_{b}<0.2$.

- In ekpyrotic type scenarios back reaction of $\mathrm{KK}$ gravitons on the evolution of spacetime is most probably not negligible. 